\title{
Evaluating patient presentations for care delivered by emergency nurse practitioners: A retrospective analysis of 12 months
}

\author{
Natasha Jennings, RN, NP, $\mathrm{MN}^{\mathrm{a}, *}$ \\ Emma Mckeown, RN, NP, MN ${ }^{a}$ \\ Gerard O'Reilly, MBBS, FACEM, MPH ${ }^{a, b}$ \\ Glenn Gardner, PhD, MEdStudies, BAppSci ${ }^{\mathrm{C}}$
}

\author{
a Faculty of Health, School - of Nursing, Queensland University of Technology, Brisbane, Queensland, \\ Australia \\ b School of Public Health and Preventive Medicine, Monash University, Melbourne, Australia \\ c The Alfred Emergency and Trauma Centre, Commercial Road, Prahran, Victoria 3004, Australia
}

Received 6 March 2013; received in revised form 17 April 2013; accepted 15 May 2013

\section{KEYWORDS}

Emergency nurse

practitioner;

Quality of care;

Fast track;

Emergency

department

\begin{abstract}
Summary
Background: The delivery of quality patients care in the emergency department (ED) is emerging as one of the most important service indicators to be measured in health services today. The emergency nurse practitioner role was implemented as a service innovation in one Melbourne, ED, Australia, in July 2004. The primary aim of the role was intended to enhance healthcare services, improve the efficiency and timely delivery of high quality care to patients.

Aim: To conduct a retrospective study of patient presentations at the ED to obtain a profile of the characteristics of patients managed by emergency nurse practitioners. Specifically the objectives of the study were to: (1) examine the demographics of the patient population and (2) evaluate data on emergency department service indicators for this patient cohort.

Method: A descriptive exploratory design was used. All patients presenting to the ED from January 01, 2011 to December 31, 2011 and managed by emergency nurse practitioners were included in the review. Data collection included baseline demographics, waiting times to be seen, length of stay, ED discharge diagnoses and referral patterns. Data were extracted and imported directly from the ED patient information system (Cerner log), for the specified time frame.
\end{abstract}

\footnotetext{
* Corresponding author. Tel.: +6139076 3405.

E-mail address: N.Jennings@alfred.org.au (N. Jennings).
}

1574-6267/\$ - see front matter @ 2013 Published by Elsevier Ltd on behalf of College of Emergency Nursing Australasia Ltd. 
Results: A total of 5212 patients were reviewed in the study period. The median age of patients was 35 years and $61 \%$ of patients were male. The most common discharge diagnosis was open wounds to hand/wrist. Waiting times to be seen by the emergency nurse practitioner were $14 \mathrm{~min}$ and length of stay for patients with a discharge disposition of home were $122 \mathrm{~min}$.

Conclusions: This study has provided information on patient baseline characteristics and performance on important service indicators for this patient sample that will inform further research to evaluate specific outcomes of the emergency nurse practitioner service.

(c) 2013 Published by Elsevier Ltd on behalf of College of Emergency Nursing Australasia Ltd.

\section{Why is this research or review needed?}

- The delivery of quality patient care in the emergency department is emerging as one of the most important service indicators to be measured in health services today.

- Research on the clinical and service characteristics of patients managed by emergency nurse practitioners is scant.

- The emergency nurse practitioner model is the fastest growing NP model in Australian with a $61 \%$ increase in the last three years.

\section{What are the key findings?}

- This paper provides a profile of the characteristics of the patient cohort managed by emergency nurse practitioners in a major urban emergency and trauma centre.

- The study provides a baseline on the performance on service indicators for patients managed by emergency nurse practitioners.

\section{How should the findings be used to influence policy/practice/research/education?}

- The challenge facing services today is to provide quality of care to emergency department patents while balancing the need for increased demand of services.

- As a rapidly expanding service innovation model there is scant local information on pattern of service and the influences on safety and quality of patient care.

- Providing baseline characteristics and results on service indicators will inform further research to evaluate specific outcomes of emergency nurse practitioner services and quality of patient care.

\section{Introduction}

Emergency departments (ED) have seen more than 7\% growth in patient presentations over the last 5 years and this has contributed to an ever-growing burden on the delivery of quality patient care. ${ }^{1}$ The capacity of EDs to consistently deliver timely, high quality patient care is impacted by the increase in the number and complexity of presentations. The increasing incidences of chronic disease in the community, reduced access to primary healthcare and fewer general practitioners have contributed to increased demand in ED services. $^{2-4}$

The delivery of quality patient care in the ED is emerging as one of the most important service indicators to be measured in health services today. The emergency nurse practitioner (E-NP) model is the fastest growing NP model nationally with a $61 \%$ increase in the last three years. ${ }^{5}$ Emergency nurse practitioners have been viewed as one potential solution to address this increased demand and overcrowding in the ED and have been employed to improve service indicators such as access and efficiency, directly impacting on quality patient care. ${ }^{6-8}$

A framework for measuring quality patient care in the ED was developed in 2011. ${ }^{9}$ The framework was formulated due to concerns that ED medical care was of varying quality and ill defined. The framework listed eight domains of quality patient care. The domains were defined as safe, effective, patient centred, timely, efficient and equitable health care to all patients. This concept has produced considerable debate within the literature, ${ }^{1,8,9}$ around the constructs of quality patient care in the ED. A universally accepted definition of quality patient care is not available as the notion is considered multi faceted and complex. ${ }^{10}$ Hence for the purposes of this review the domain referring to timely patient care will be examined. Patients, clinicians, administrators and policy makers use time performance indicators as a measure of service effectiveness and efficiency. Although, there is limited evidence on the effectiveness of E-NP on ED service outcomes and the quality of patient care. Therefore the purpose of this research is to evaluate E-NP service indicators as a measure of quality patient care. This descriptive exploratory review will:

1. Examine the demographic and clinical characteristics of the patient population.

2. Evaluate ED service indicators for this cohort including:

- Waiting time.

- Overall length of stay in the emergency department for both admitted and discharged patients.

- Disposition diagnosis and destination.

\section{Design}

A descriptive exploratory design utilising a sampling frame of 12 months from 1st January 2011 to 31st December 2011 was used. All patients managed by the E-NPs during the study period were included in the review. 


\section{Sample/participants}

The study was conducted in an Emergency Department in Melbourne, Australia. The ED is an urban, adult tertiary department with an annual ED census in 2011 of over 56,000 patients. The ED model of care is serviced by a traditional medical service with treatment decisions carried out by medical practitioners. This model also incorporates the provision of E-NP services. Emergency nurse practitioners are a hybrid service delivery model, holding both nursing and medical skills with an emphasis on health promotion, education and holistic care. ${ }^{6}$ The E-NP model of care specifically includes assessment and management of patients using critical decision-making skills, referring directly to other health care providers, prescribing medications, performing interventions and ordering and interpreting diagnostic investigations. Emergency nurse practitioners' work both independently in managing patients and collaboratively within the ED model of care. Emergency nurse practitioners are well established in this setting since their inception in 2004 with eight E-NPs providing seven days a week service from $07: 00 \mathrm{pm}$ to $23: 30 \mathrm{pm}$. The emergency nurse practitioner staffing profile during the study period included two fulltime E-NP, two part time E-NP candidates, two E-NPs on maternity leave and 2 part time E-NP's. The effective full time hours employed at the site was a total of five. Emergency nurse practitioners' individual patient workloads on average are 8-10 patients per shift. Dependent upon the breadth of experience or whether the E-NP is undertaking their candidate training programme often impacts the numbers of patients seen. The emergency nurse practitioner service also includes collaborative practice, so in fact the E-NP may have signed on and treated for example eight patients on their shift but they may have also contributed care to patients in trauma and resuscitation for a proportion of their shift time. The emergency nurse practitioner service is geographically located in the fast track area of the ED. Fast track services enhance ED care processes in an area designated for timely assessment, treatment and discharge of patients seeking primary care type services for less serious illnesses and injuries. ${ }^{11,12}$ The emergency nurse practitioner focuses on the management of patient presentations within specific inclusion criteria. Patient presentations managed include cellulitis, open wounds, limb injuries, abdominal or back pains and other minor presentations.

\section{Inclusion criteria}

This study was a retrospective review of all patients managed by E-NPs at the study site during the sampling time frame. All study patients must have presented to the ED between the hours of 07:00 h and 23:00 h when E-NP service was available. Patients whose disposition diagnosis was determined as "left after clinical advice"' or "left at own risk" 'were excluded from the study, as they were not managed by the E-NP service and left the ED without any clinical management.

\section{Data collection}

All review data were entered on the ED patient information system (Cerner Log), a patient attendance registry that has been used at the site for 14 years. The log is a computerised system that collects surnames of individual practitioners that manage ED patient care.

\section{Ethical considerations}

The study was approved by the Hospital Research Ethics committee as a low risk project in June 2012.

\section{Data analysis}

The ED service indicators examined included waiting and length of stay (LOS) times for patients managed by E-NPs. Waiting time was defined as time in minutes from initial registration until treatment commencement by the E-NP. Length of stay was defined as the time in minutes the patient spent in the ED from initial registration until time of disposition from the ED. The Victorian Department of Health Services mandated emergency department LOS targets until the end of the calendar year of 2011. Compliance with the $4 \mathrm{~h}$ ED LOS for at least $80 \%$ of non-admitted patients was anticipated. Currently, the proportion of non-admitted patients with a length of stay under $4 \mathrm{~h}$ nationally in major metropolitan hospitals is $54 \% .^{13}$

Disposition was defined as the final destination of the patient's acute care episode. A disposition diagnosis was based upon the common diagnostic subgroup classifications of ICD-10-AM (Statistical classification of Diseases and Related Health Problems, 10th Revision, Australian Modification).

All patients were de-identified prior to data analysis. Descriptive statistics were used to summarise the profiles of ED presentations for E-NP managed care. Data were analysed using Stata (StataCorp., College Station, TX, USA). Data were not normally distributed so medians and interquartile ranges (IQR) ranges are presented for analysis.

\section{Results}

A total of 5357 patients were managed by E-NPs between January 01, 2011 and December 31, 2011. One hundred and forty-five patients were excluded from the analysis as their disposition diagnosis included "left after clinical advice" or "left at own risk" (see Table 1). The median age of patients was 35 years $(n=5212)$ and $61 \%$ of patients were male.

The most common patient presentations managed by the E-NP service were open wounds to wrist or hand $(n=547)$. The next most common presentations included fracture of and unspecified parts of the wrist and hand $(n=292)$, Surgical follow-up care unspecified $(n=244)$ and sprain/strain of ankle unspecified $(n=202)$, respectively. A list of the top 10 ICD discharge diagnoses is provided as Table 2. In total there were 359 different discharge diagnoses described by the E-NP service model.

Median waiting times to be seen by the E-NP was 14 min (IQR 7-33) and length of stay for patients with a discharge 
Table 1 NP patient demographics.

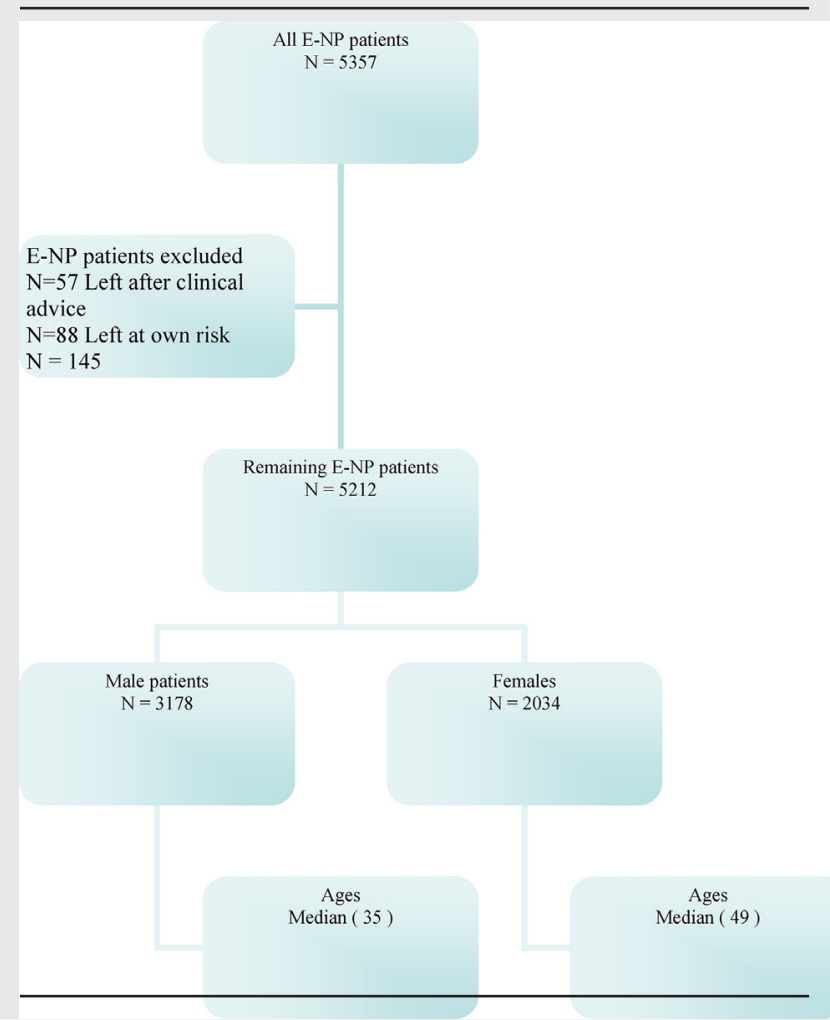

disposition of home was $122 \mathrm{~min}$ (IQR 77-177). Those patients whom discharge disposition was admission/other, their length of stay was 271 min (IQR 190-405) (see Table 3). Overall, $95.9 \%$ of the discharged home group $(n=4509)$ left the ED within the 4-h service target.

The most common discharge disposition was home $(n=4509)$. The next most common discharge disposition was ward $(n=355)$ and short stay unit $(n=252)$ (see Table 4$)$. Patients were most commonly referred to their Local Medical Officer $(n=85 \%)$.
Table 3 Waiting times and length of stay for discharge home.

\begin{tabular}{ll}
\hline Service indicators & E-NP service (minutes) \\
\hline Waiting times & 14 (IQR 7-33) \\
Length of stay & 131 (IQR 82-200) \\
\hline
\end{tabular}

Table 4 Disposition.

\begin{tabular}{lrr}
\hline Disposition & $\mathrm{N}=$ & $\%$ \\
\hline Home & 4509 & 86.5 \\
Left at own risk after treatment started & 25 & 0.5 \\
Short stay unit admit & 252 & 4.8 \\
Theatre & 47 & 0.9 \\
Ward admit & 355 & 6.8 \\
Other & 24 & 0.5 \\
Total= & 5212 & 100 \\
\hline
\end{tabular}

\section{Discussion}

The purpose of this study was to profile the characteristics of patients managed by E-NPs and to evaluate specific service indicators as a measure of quality patient care. This review is timely as part of the National Health Reform agenda will see the roll out of the new National Access Target for Emergency Departments, known as the 'National Emergency Access Target' ('NEAT') in 2012. The NEAT is measured as the percentage of patients who leave the ED within $4 \mathrm{~h}$ of their arrival. The time is measured from when the patient arrives at the ED to the time the patient has been discharged to either admission to a ward, transferred to another hospital, or goes home. The initial compliance target for 2012 is mandated at $72 \%$ with expectations of that figure gradually increasing to $90 \%$ by the year $2015 .{ }^{14}$ During the sampling time frame the Victorian Department of Health Services had mandated targets for time target compliances and NEAT did not come into affect until after the study period.

Table 2 Top 10 patient presentations by ICD codes.

\begin{tabular}{lrll}
\hline Ranking & $N=$ & ICD code & Descriptors \\
\hline 1 & 547 & S619 & Open wound of wrist and hand part, part unspecified \\
2 & 292 & S628 & Fracture of other and unspecified parts of wrist and hand \\
3 & 244 & Z489 & Surgical follow-up care, unspecified \\
4 & 202 & S9340 & Sprain and strain of ankle, part unspecified \\
5 & 178 & L039 & Cellulitis, unspecified \\
6 & 144 & S0188 & Open wound of other parts of head \\
7 & 136 & Z209 & Contact with and exposure to unspecified communicable disease \\
8 & 109 & S929 & Fracture of foot, unspecified \\
9 & 101 & Z099 & Follow-up examination after unspecified treatment for other conditions \\
10 & 99 & S936 & Sprain and strain of other and unspecified parts of foot \\
TOTAL & 2052 & 359 & \\
\hline
\end{tabular}


Health services today are focusing on quality of patient care in the ED and the ability to deliver timely and efficient healthcare to all patents. The recently implemented NEAT targets applied in 2012 have forced health services to reevaluate their delivery of care and evaluate their service models. Time based performance targets such as wait times and LOS measures are utilised nationally to compare and contrast health service efficiency. In this review large numbers of patients were managed by the E-NP service with the NEAT target, with the most common discharge diagnosis of open wounds to wrist or hand and fractures to the wrist or hand.

\section{Demographics}

The median ages of patients managed by E-NPs were 35 years with $61 \%$ were male. Considering E-NP services tend to be based in lower acuity areas and manage large numbers of minor injuries, the median age and gender profile was consistent with other published studies. ${ }^{15}$ Typically males in there thirties make up the majority of the population performing labouring and working with machinery occupations. These high-risk occupations are considered to sustain more injuries requiring presentations to the ED for management. The resultant care is managed by the E-NP services. In the Australian context of studies undertaken in EDs and fast tracks, males were more than $55 \%$ of the population with median ages of around 30 years. ${ }^{12,16-18}$ In the UK, males were also identified as the majority of presentations managed by E-NP services ${ }^{19,20}$ and one randomised controlled trial of nurse practitioner services, ${ }^{21}$ saw males contributing the majority of patients at $58 \%$ and the median age of 37 years.

\section{Wait times}

Emergency nurse practitioner services are generally focused on patient presentations within Australasian Triage Scale (ATS) categories 2-5. ${ }^{22}$ The ATS is designed for use in hospital based emergency services throughout Australia and New Zealand. The ATS scale is utilised for rating clinical urgency and attempts to ensure patients are seen in a timely manner commensurate with their medical urgency. Emergency nurse practitioner patients therefore can wait from $10 \mathrm{~min}$ to $120 \mathrm{~min}$ for treatment in the ED. The median wait time for patients managed by E-NPs was $14 \mathrm{~min}$ (IQR 7-33) and all patients in this study were seen within the 120 min time frame. Although wait times were not stratified according to ATS categories it is anticipated if waiting times were delayed this would have a negative impact on the service indicator of length of stay. In this study length of stay was very favourable and not affected by any measures of wait times. The median waiting time for fasttrack patients was 50 min (IQR 23-96). Another similar local urban $\mathrm{ED},{ }^{23}$ saw nurse practitioners proportion of patients seen within ATS recommendations reaching levels of $82.5 \%$. Other Australian results for waiting times range from median of 13 min (IQR 6-28), ${ }^{18,24}$ and in New Zealand ${ }^{14} 14$ min (IQR 527).

\section{Length of stay}

The median LOS managed by E-NPs was 131 min (IQR 82-200) for those patients in the study whose discharge disposition was 'home'. Comparisons of LOS can be made with the literature at both an Australian and international context. One Australian transitional E-NP models median LOS was $109 \mathrm{~min}^{18}$ and in the New Zealand setting ${ }^{19}$ E-NP LOS was $117 \mathrm{~min}$, and other local models E-NP LOS was $102 \mathrm{~min}, 23,24$ which utilise the same definition for LOS outcomes. International comparisons of E-NP LOS include 102 min $^{20,25}$ from Ireland and the United Kingdom with E-NP LOS 83 min. ED LOS compliance with the $4 \mathrm{~h}$ non-admitted patient target in this study was $95.1 \%$, very consistent with local results ${ }^{17}$ showing a compliance of $95.9 \%$.

\section{Presentation types}

In the study setting E-NP services were located geographically in fast track and managed patients within a defined scope of practice. The aim of the fast track service is to provide an efficient and effective way of caring for patients who present to the ED with minor illnesses and injuries. There is strong evidence to suggest that fast track services streamline the management of patients impacting favourably upon patient outcomes. ${ }^{26-28}$ Fast track patients can be directly managed rapidly and do not require intensive concentrated ED care. The most common presenting discharge diagnoses were closely related to open wounds (hand/wrist), sprains/strains (foot/ankle/hand/wrist) and fractures (hand/wrist). Other Australian E-NP studies ${ }^{18,23}$ have also consistently concentrated on musculoskeletal/soft tissue group with the most common diagnoses of open wound (hand/wrist) and fracture (hand/wrist).

\section{Referral patterns}

The cohort of patients seen by E-NPs most often require follow up and re assessment of their antibiotics compliance and safety, dressings, and follow up of results. Therefore the most common referrals made by E-NPs were to the local medical officer. The organisation training for the E-NP service requires the practitioner to provide material and resources for all patients being discharged home to their local medical officer. Previous research has demonstrated patients receive more health information and better discharge instructions when compared with traditional care. ${ }^{29-32}$

Emergency nurse practitioners are a hybrid service delivery model, holding both nursing and medical skills with an emphasis on health promotion, education and holistic care. The challenge facing health services today is to provide quality of care to ED patients while balancing the complex need for increased demand of services. Emergency nurse practitioners are one potential innovation to address this need. Research has shown that this service innovation has been rapidly adopted in Australia and internationally but there is scant information to date on the pattern of service and the influence on safety and quality of patient care. 


\section{Limitations}

This study drew upon a large database to describe the demographic and clinical features of a cohort of patients whose ED presentation was managed by E-NP service. However, consideration of these study findings needs to take into account some limitations in the study design. With a retrospective study design, there is the likelihood that some of the data collection was incomplete and inaccurate. Furthermore we limited the variables to those where data was most likely to be complete; other unmeasured variables may have contributed to a more comprehensive evaluation of waiting times and length of stay. The study was conducted at a single site with a well-established E-NP service. Hence the utility of the study's findings is limited to service contexts with similar characteristics. The study does however provide a substantial baseline dataset to inform subsequent prospective research into the clinical efficacy and service effectiveness of the E-NP service innovation.

\section{Conclusions}

This research into E-NP service has illustrated that patient care is delivered to a large number of patients with consistent demography as interpreted from the existing literature. It has provided baseline characteristics and results on service indicators that will inform further research to evaluate specific outcomes of the E-NP service and quality of patient care.

\section{Author contributions}

N.J., G.G. conceived and designed the study. N.J., E.M. developed the study protocol and supervised data collection. N.J., E.M., G.O., and G.G. analysed the data. All authors contributed and approved the manuscript.

\section{Provenance and conflict of interest}

None of the authors are associated with provenance or conflicts of interests in regards to the manuscript. This paper was not commissioned.

\section{Funding statement}

This research received no specific grant from any funding agency in the public, commercial, or not-for-profit sectors.

\section{Ethics approval}

The study was approved by the Hospital Research Ethics committee as a low risk project in June 2012.

\section{References}

1. Lowthian J, Cameron P. Editorial. Improving timeliness while improving the quality of emergency department care. Emerg Med Australas 2012;24:219-21.
2. Australian Health Workforce Association. http://www. ahwo.gov.au/documents/NHWT/The\%20health\%20workforce\% 20in\%20Australia\%20and\%20factors\%20influencing\%20current\% 20shortages.pdf; [accessed 27.03.12].

3. Australian Institute of health and welfare. http://www.aihw.gov.au/sqhc-definitions/; 2009 [accessed 26.11.12].

4. Sprivulis P, da Silva J, Jacobs I, Jelinek G, Swift R. ECHO: the Western Australian emergency care hospitalisation and outcome linked data project. Aust NZ J Public Health 2006;30(2): 123-7.

5. Lowthian J, Curtis A, Cameron P, Stoelwinder J, Cooke M, McNeil $J$. Systematic review of trends in emergency department attendances: an Australian perspective. Emerg Med J 2011;28:373, http://dx.doi.org/10.1136/emj.2010.099226.

6. Middleton S, Gardner A, Gardner G, Della P. The status of Australian nurse practitioners: the second national census. Aust Health Rev 2011;35(4):448-54.

7. Wilson $\mathrm{K}$, Cameron $\mathrm{P}$, Jennings $\mathrm{N}$. Emergency nurse practitioners: an underestimated addition to the emergency care team. Emerg Med Australas 2008;20(6):453-5.

8. O'Connell J, Gardner G. Clinical competencies for emergency nurse practitioners. Australas Emerg Nurs J 2012;15(4):185-252, http://dx.doi.org/10.1016/j.aenj.2012.10.004.

9. Cameron PA, Schull MJ, Cooke MW. A framework for measuring quality in the emergency department. Emerg Med J 2011;28(9):735-40, http://dx.doi.org/10.1136/emj.201.112250.

10. Bennett $P$. Nursing quality indicators: the next step in enhancing quality in emergency care. Int Emerg Nurs 2012;20(3):179-86, http://dx.doi.org/10.1016/j.ienj.2012.04.001.

11. State Government of Victoria, Australia, Department of Health. http://www.health.vic.gov.au/emergency/fasttrack discussion_paper.pdf; [accessed 04.02.13].

12. State Government of Victoria, Australia, Department of Health. http://www. health.vic.gov.au/emergency/streamingcare0109. pdf; [accessed 04.02.13].

13. National Health Performance Authority. http://www.nhpa.gov. au/internet/nhpa/publishing.nsf/Content/Media-Release-

First-report-shows-large-variation-in-hospital-performance; [accessed 26.11.13].

14. Australian Government, Department of health and Ageing. Expert Panel Review of Elective Surgery and Emergency Access Targets under the National Partnership Agreement on Improving Public Hospital Services. http:// www.yourhealth.gov.au/internet/yourhealth/publishing.nsf/ Content/Expert-Panel-Report-Section-3; [accessed 03.01.13].

15. Dinh $M$, Walker A, Parameswaran A, Enright $\mathrm{n}$. Evaluating the quality of care delivered by an emergency department fast track unit with both nurse practitioners and doctors. Australas Emerg Nurs J 2012, http://dx.doi.org/10.1016/j.aenj.2012.09.001.

16. Victorian Government Health Information. Victorian hospital data reports. Reference files. Victorian Emergency Minimum Dataset - VEMD. http://www.health.vic.gov.au/ archive/archive2011/hosdata/datafields.htm; 2013 [accessed 26.11.12].

17. Lee $G$, Jennings N. A comparative study of patients who did not wait for treatment and those treated by Emergency Nurse practitioners. Australas Emerg Nurs J 2006;9:179-85.

18. Considine J, Kropman M, Stergiou HE. Effect of clinician designation on emergency department fast track performance. Emerg Med J 2010;27(11):838-42.

19. Fry M, Fong J, Asha S, Arendts G. A 12-month evaluation of the impact of transitional emergency nurse practitioners in one metropolitan emergency department. Aust Emerg Nurs $J$ 2011;14:4-8, http://dx.doi.org/10.1016/j.aenj.2010.10.001. 
20. Colligan M, Collins C, Foley B, Jones P, Miles J, Zeng I. Emergency nurse practitioners: do they provide an effective service in managing minor injuries, compared to emergency medicine registrars? NZ Med J 2011;124 http://journal.nzma.org.nz/journal/124-1344/4907/

21. Thompson W, Meskell P. 920120. Evaluation of an advanced nurse practitioner (emergency care) - an Irish perspective. J Nurs Pract 2013;8(3), http://dx.doi.org/10.1016/j.nurpra.2011.09.002.

22. Sakr M, Angus J, Perrin J, Nicholl C, Nixon J, Waldrope J. Care of minor injuries by emergency nurse practitioners or junior doctors: a randomised controlled trial. Lancet 1999;354(9187):1321-6.

23. Australian college of emergency medicine: policy on the Australasian triage scale. http://www.acem.org.au/ media/policies_and_guidelines/P06_Aust_Triage_Scale_-_Nov_ 2000.pdf; [accessed 22.5.12].

24. Considine J, Martin R, Smit D, Winter C, Jenkins J. Emergency nurse practitioner care and emergency department patient flow: case-control study. Emerg Med Australas 2006; 18(4):385-90.

25. Jennings $N$, O'Reilly G, Lee G, Cameron P, Free B, Bailey M. Evaluating outcomes of the emergency nurse practitioner role in a major urban emergency department, Melbourne, Australia. J Clin Nurs 2008;17(8):1044-50, http://dx.doi.org/10.1111/j.1365-2702.2007.02038.x.
26. Byrne G, Richardson M, Brunsdon J, Patel A. An evaluation of the care of patients with minor injuries in emergency settings. Accid Emerg Nurs 2000;8:101-9.

27. Kelly $A M$, Bryant $M$, Cox L, Jolley D. Improving emergency department efficiency by patient streaming to outcomes-based teams. Aust Health Rev 2007;31(1): $16-21$.

28. Kwa P, Blake D. Fast track: has it changed patient care in the emergency department? Emerg Med Australas 2008;20:11-3, 26.

29. Carter A, Chochinov A. A systematic review of the impact of nurse practitioners on cost, quality of care, satisfaction and wait times in the emergency department. Can J Emerg Med 2007;9(4):286-95.

30. Change E, Daly J, Hawkins A, McGirr J, Fielding K, Hemmings $L$, et al. An evaluation of the nurse practitioner role in a major rural emergency department. J Adv Nurs 1997;30: 260-8.

31. Cooper M, Lindsay G, Kinn S. Evaluating emergency nurse practitioner services: a randomised controlled trial. J Adv Nurs 2002;40:721-30.

32. Lutze M, Ratchford A, Fry M. A review of the transitional emergency nurse practitioner. Australas Emerg Nurs J 2011;14:226-31, http://dx.doi.org/10.1016/j.aenj.2011.06.003. 